\title{
Electrical characterization of p-GeSn/n-Ge diodes with interface traps under dc and ac regimes
}

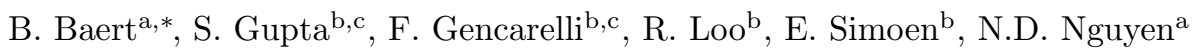 \\ ${ }^{a}$ Department of Physics, Solid State Physics - Interfaces and Nanostructures, University of Liege, 4000 Liege, Belgium. \\ ${ }^{b}$ IMEC, Kapeldreef 75, 3001 Leuven, Belgium \\ ${ }^{c}$ MTM department, KU Leuven, Kasteelpark Arenberg 10, 3001 Leuven, Belgium
}

\begin{abstract}
In this work, the electrical properties of p-GeSn/n-Ge diodes are investigated in order to assess the impact of defects at the interface between Ge and GeSn using temperature-dependent current-voltage and capacitance-voltage measurements. These structures are made from GeSn epitaxial layers grown by CVD on Ge with in-situ doping by Boron. As results, an average ideality factor of 1.2 has been determined and an activation energy comprised between $0.28 \mathrm{eV}$ and $0.30 \mathrm{eV}$ has been extracted from the temperature dependence of the reverse-bias current. Based on the comparison with numerical results obtained from device simulations, we explain this activation energy by the presence of traps located near the GeSn/Ge interface.
\end{abstract}

Keywords: GeSn, diodes, electrical characterization, I-V characteristics, C-V characteristics, numerical simulation, impedance spectroscopy, admittance spectroscopy

\section{Introduction}

Germanium-tin is a very promising semiconducting alloy with many attractive properties that are of interest for various applications. One of them is a direct band-gap, which is expected to occur for Sn concentrations around $10 \%$ in fully relaxed GeSn layers. This is therefore particularly useful in view of developing integrated, Si-compatible, photodiodes for long wavelength applications[1-3]. Another advantage of GeSn is the use as stressor or as strained material, resulting from the lattice mismatch with Ge or Si. It is therefore a good candidate as compressive source and drain stressor in p-type Ge-channel metal oxide semiconductor field effect transistors (pMOSFETs) [4-7] or as channel material $[8,9]$ within a postscaling approach[10]. Being a group-IV material is also an asset for the integration into current Si-based technologies, although the successful incorporation of other alloys such as III-V compounds on Si has already been demonstrated[11-14].

For such applications, however, few electrical investigations of these materials have been reported. The effect of defects at or near the interface between Ge and GeSn, especially, is not completely characterized. To this perspective, diodes are very useful for the characterization of future complete MOSFETs, as their process flows contain many of the steps necessary to the fabrication of a full MOSFET device. We therefore investigated the electrical characteristics of $\mathrm{GeSn} / \mathrm{Ge}$ diodes in view of assessing the

\footnotetext{
* Corresponding author

Email address: Bruno.Baert@ulg.ac.be. (B. Baert)
}

influence of defects in such materials. We endeavoured to determine their activation energy and calculated the ideality factor of the diodes from current-voltage characteristics. We also performed capacitance-voltage measurements to determine the carrier concentration of the Ge substrate and to assess the impact of the defects on the frequency dispersion.

\section{Method}

\subsection{Devices}

A $200 \mathrm{~nm}$ thick Boron doped $\mathrm{p}+\mathrm{GeSn}$ layer was grown on top of a $4^{\prime \prime}, 450 \mu \mathrm{m}$ thick, Ge substrate by chemical vapor deposition (CVD) at $320^{\circ} \mathrm{C}[15,16]$. Blanket n-doped $\mathrm{Ge}(100)$ wafers with low $\left(\sim 10^{16} \mathrm{~cm}^{-3}\right)$ carrier concentration were used as substrates. The GeSn layer is in-situ doped by adding $\mathrm{B}_{2} \mathrm{H}_{6}$ to the $\mathrm{Ge}_{2} \mathrm{H}_{6}$ and $\mathrm{SnCl}_{4}$ precursors with a Boron target concentration of $3 \times 10^{18} \mathrm{~cm}^{-3}$. This target value is experimentally confirmed by secondary ion mass spectroscopy (SIMS) measurements and, although $\mathrm{B}$ activation has not been measured on this specific sample, $100 \%$ B activation has been reported for similar GeSn layers[15]. According to (224) reciprocal space mappings (RSM) obtained from X-ray diffraction (XRD) measurements, the GeSn strain relaxation level is $43 \%$. From the in-plane and out-of-plane lattice parameters measured by (224)RSM XRD, using a corrected Vegard's law, the substitional Sn content in these layers is determined to be $5.8 \%[17]$. Al contacts with a thickness of $100 \mathrm{~nm}$ have subsequently been deposited. 
An Agilent 4156 Semiconductor Parameter Analyzer, a Keithley 2400 Source Meter and an HP4192A LF Impedance Analyzer were used for the measurements of these diodes. For the frequency measurements, bias oscillation with a $50 \mathrm{mV}$ amplitude has been applied. Measurements as a function of diode temperature have been performed using a liquid nitrogen cryostat. The temperature variation is based on positioning the sample above a Dewar bottle filled with liquid nitrogen. The resulting vertical gradient of temperature between the temperature of liquid nitrogen $(77 \mathrm{~K})$ and room temperature allows to access a wide range of temperatures through computer-controlled feedback and positioning of the sample with a precision of 0.1 K.

\subsection{Numerical simulations}

A home-built numerical tool based on the numerical solution of the basic semiconductor equations has subsequently been used to model the diode structure[18, 19]. These are applied to a unidimensional structure comprising one or several semiconducting layers sandwiched between two metallic electrodes. Therefore, all quantities depend only on one spatial variable $x$. Defects states, acting as traps with a specific energy within the band gap and a total concentration $N_{t}$ which can be varied along the $x$ position, have been considered.

The physics of the junction is governed by the classical equations for the electrostatic potential $\Psi$, the electron and hole concentrations $n$ and $p$, and the concentrations of occupied defect states $n_{t}[19]$. The electric potential $\Psi$ obeys Poisson's equation

$$
\epsilon \frac{\mathrm{d}^{2}}{\mathrm{~d} x^{2}} \Psi=-q\left(p-n+N_{D}-N_{A}+C_{t}\right),
$$

where $N_{D}$ and $N_{A}$ are the shallow donor and acceptor concentrations, which are assumed to be fully ionized, and $C_{t}$ is the trap charge concentration. $C_{t}$ equals $-n_{t}$ for acceptor states and equals $\left(N_{t}-n_{t}\right)$ for donor states, $q$ is the electronic charge. The continuity equations for electrons, holes and occupied defect states are

$$
\begin{gathered}
\frac{\partial n}{\partial t}=\frac{1}{q} \frac{d}{d x} J_{n}-R_{b b}-R_{n t} \\
\frac{\partial p}{\partial t}=-\frac{1}{q} \frac{d}{d x} J_{p}-R_{b b}-R_{p t} \\
\frac{\partial n_{t}}{\partial t}=R_{n t}-R_{p t}
\end{gathered}
$$

In these equations, $J_{n}$ and $J_{p}$ are the electron and hole current densities. They are given by the sum of a drift and a diffusion term, i.e.

$$
J_{n}=q n \mu_{n} E+q D_{n} \nabla n
$$

where $E$ is the electric field, $\mu_{n}$ is the electron mobility and $D_{n}$ is the diffusion constant. Einstein relations between $\mu_{n}$ and $D_{n}$ are assumed. A similar expression holds for the hole current density $J_{p}$.

The $R_{b b}$ term accounts for direct band to band recombination processes and $R_{n t}$ and $R_{p t}$ are, respectively, the total electron transition rates from the conduction band to trap states and from trap states to the valence band[20]. Each defect level is supposed to interact independently with the valence band and the conduction band. The interaction between trap states is neglected. Complementary details on the numerical procedure can be found in Refs.[18-23].

The system is solved under steady-state conditions first, corresponding to the application of a dc bias to the diodes. Starting from this steady-state solution, resolution under small-signal regime is subsequently performed, analogous to applying a small ac bias. We obtain, as results of the simulations, microscopic quantities such as the spatial distribution of carrier concentrations, electric potential and electric field and electron and hole current densities. Macroscopic quantities like the total steady-state current across the structure, the small-signal conductance $G$ and capacitance $C$ (calculated from the complex admittance $Y=\tilde{J} / \tilde{V})$, are also obtained for various sets of physical parameters, e.g. the dopant concentrations and band-gap energies of the materials. Simulations therefore allow us to observe the effect of the variation of physical parameters on those quantities and give an interpretation of the experimental measurements by establishing physical links between the macroscopic and microscopic quantities.

Calculations have first been performed using estimated values of the carrier concentrations, band-gap energy and defect densities. Band-gap energies of $0.67 \mathrm{eV}$ and 0.58 $\mathrm{eV}$ have been used for the Ge substrate and GeSn layer, respectively[24]. These parameter values have subsequently been modified in order to match the measurements within a feedback process between experiment and simulations.

\section{Results and discussion}

\subsection{Room-temperature current-voltage characteristics}

In order to assess the presence of traps in the electrical properties of the diodes, we have first investigated the current-voltage (I-V) characteristics at room temperature and in the forward-bias regime (Fig. 1 (a)). The ideality factor $n$ has been extracted from diodes with different contact diameters. Biases restricted to a range from $0 \mathrm{~V}$ up to $0.25 \mathrm{~V}$ have been used, in order to avoid the effect of the series resistance of the Ge substrate, which becomes prominent for higher biases. Values of $n$ comprised between 1.2 and 1.3 are obtained, depending on the contact diameter of the diode. The larger value is associated to the diode with the larger contact diameter of $600 \mu \mathrm{m}$, whereas all other diameters between $120 \mu \mathrm{m}$ and $300 \mu \mathrm{m}$ result in a value of $n$ between 1.2 and 1.22. As the total current across the structure is the highest for this larger diameter, current limitation due to the high resistivity of the $450 \mu \mathrm{m}$ 

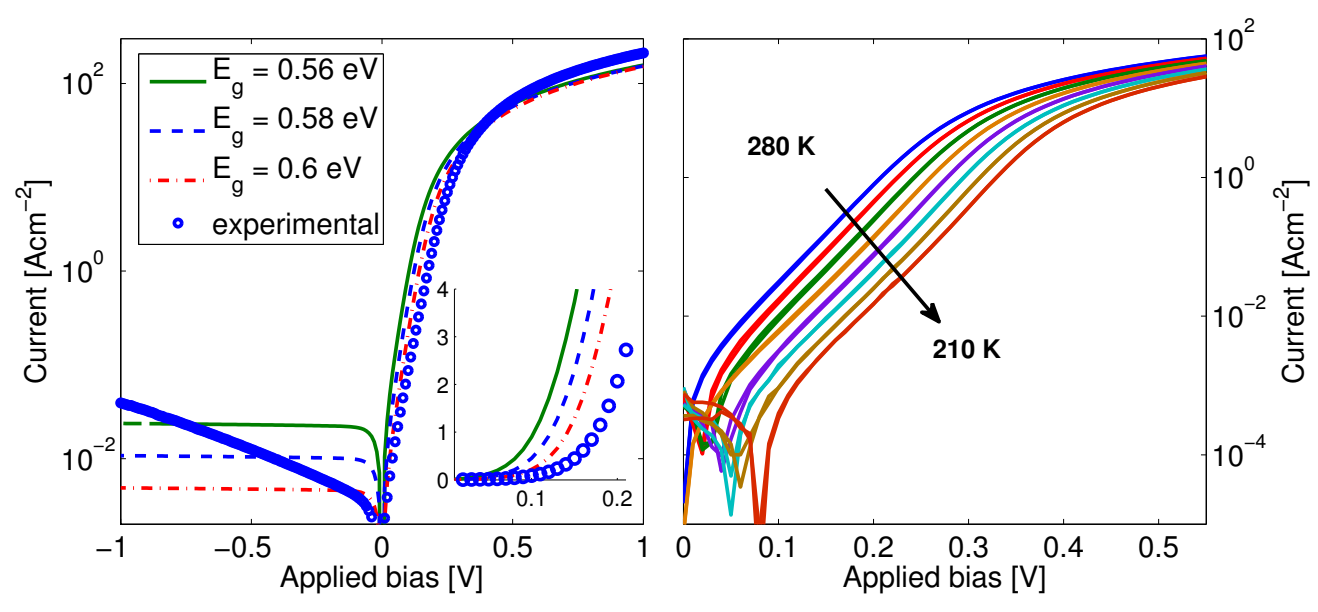

Figure 1: (a) Room-temperature, experimental and simulated, I-V curves of the p-GeSn/n-Ge diodes (for three different GeSn bandgap energies : $0.56 \mathrm{eV}, 0.58 \mathrm{eV}$ and $0.6 \mathrm{eV}$ ). (b) Experimental forward I-V curves for temperatures between $280 \mathrm{~K}$ and $210 \mathrm{~K}$.

thick weakly doped substrate could explain the observed difference in the resulting ideality factor. In order to avoid this occurrence, the bias range within which the ideality factor was extracted is such that the value of the coefficient of determination $R^{2}$ of the linear fit is kept above 0.99. However, a slight difference in the slope of the linear fit for this larger contact area, due to the onset of current limitation, though not sufficient to degrade the $R^{2}$ value, could lead to the observed difference of $n$ values as compared with the other diameters. Overall, the mean value of 1.2 for the ideality factor therefore is a clear indication of a close-to-ideal diode.

Nevertheless, as an ideality factor $n$ greater than unity is expected to indicate the presence of recombination processes in the diodes, simulations including Shockley-ReadHall (SRH) traps in the GeSn layer have therefore been considered. These traps are expected to account for the presence of defects in the crystalline structure of the semiconducting materials.

The effect of traps, as well as that of a change in the value of the GeSn layer band-gap, can be observed in Fig. 2, where the ideality factor obtained from simulations is shown as a function of trap concentration for various GeSn band-gap energies. The ideality factor increases with the trap concentration, as expected from their role as recombination centers in the depletion region. For a given trap concentration, the ideality factor increases when the GeSn band-gap decreases. This behaviour can also be explained by the improved recombinations due to the smaller energy separation between the conduction and valence bands. As the bandgap energy of the GeSn epilayer is supposed to be around $0.58 \mathrm{eV}$ for the $\mathrm{Sn}$ concentration of these diodes[25], the calculated ideality factor close to 1.2 would correspond to trap concentrations in the GeSn layer comprised between $5 \times 10^{17} \mathrm{~cm}^{-3}$ and $2 \times 10^{18} \mathrm{~cm}^{-3}$.

The I-V characteristics measured at room temperature show a ratio of $10^{4}$ between the current a $+1 \mathrm{~V}$ and $-1 \mathrm{~V}$ bias. Simulations of the current across a similar structure as the experimental one (see Fig. 1) show a matching behaviour in the forward bias regime. In reverse bias, though, the measured reverse saturation current increases much faster than in the simulations with the initial set of parameters. Variation of the GeSn band-gap energy, which is related to the Sn concentration, shows that the reverse saturation current increases when the band-gap decreases (i.e. the $\mathrm{Sn}$ concentration increases). This can be linked to the intrinsic carrier concentration $n_{i}$ that increases exponentially when the band-gap energy decreases. Even though the simulated reverse saturation current increases when the band-gap energy is lowered, the increase rate as a function of applied reverse bias remains at best linear, whereas the growth of the experimental reverse current is almost exponential. Increasing the trap concentration in the GeSn layer in the simulations also gives rise to an increased reverse current, but not to the extent observed in the experimental data.

\subsection{Temperature-dependent current-voltage characteristics}

In order to further understand the mechanism at the origin of the observed reverse current, I-V characteristics as a function of diode temperature have been measured. Such characteristics allow to determine activation energies $E_{a}$ from the reverse saturation current $I_{S}$. Indeed, $I_{S}$ can be expressed as being proportional to $e^{\left(-E_{a} / k T\right)}$, where $k$ is the Boltzmann constant and $T$ is the absolute temperature. From an Arrhenius plot of $I_{S}(T)$ as a function of $1 / k T$, the activation energy $E_{a}$ can be extracted[26-28]. This activation energy can be associated to an energy barrier that has to be overcome for the corresponding process to occur.

From the first linear part at small forward bias of the I$\mathrm{V}$ characteristics, the ideality factor $n$ and reverse current 


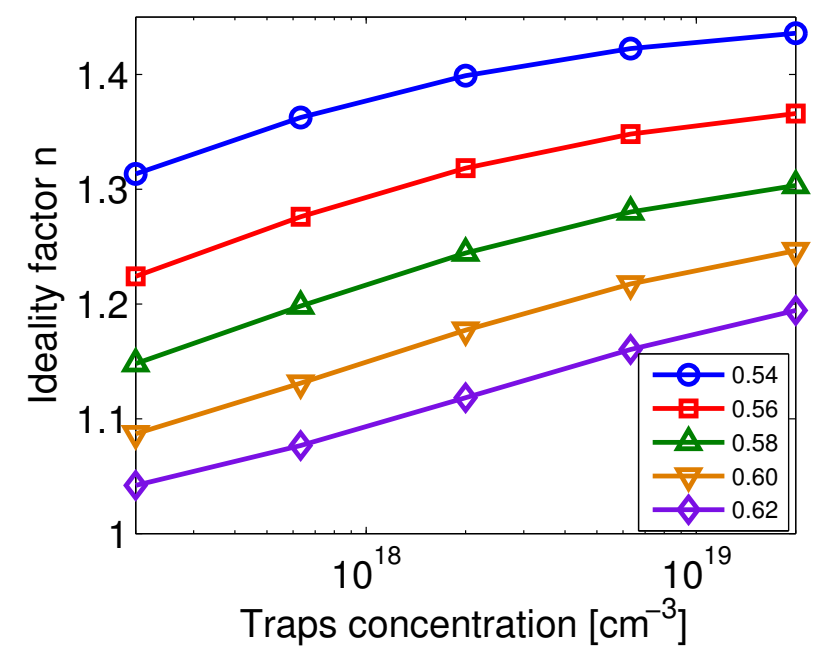

Figure 2: Ideality factor $n$ from simulations as a function of trap concentration for GeSn band-gap energies between 0.54 eV and 0.62 eV (values indicated in the inset) and a Ge substrate dopant concentration of $1.5 \times 10^{16} \mathrm{~cm}^{-3}$.

$I_{S}$ are extracted at various temperatures[29]. The slope of the Arrhenius plot of $I_{S}$ as a function of $1 / T$, shown in Fig. 3, yields an activation energy comprised between $0.28 \mathrm{eV}$ and $0.30 \mathrm{eV}$. The linearity of this Arrhenius plot is relatively good and the measurements are reproducible within the confidence interval given above.

Simulations of the same Arrhenius plot, initially without considering defects in the structure, yield an activation energy of $0.6 \mathrm{eV}$, close to the value of the band-gap of the GeSn material. Variation of the GeSn band-gap value in the simulations induces a corresponding variation of the calculated activation energy, whereas variation of the bandgap of the Ge layer induces no such variation of the calculated activation energy, which remains at a value corresponding to the GeSn band-gap energy. This result clearly indicates that the activation energy is indeed linked to the band-gap of GeSn.

Adding traps into the simulations shows no difference in the obtained activation energy when these traps are located in the GeSn layer. When these traps are located in the Ge layer, though, the obtained activation energy is closer to $0.3 \mathrm{eV}$. Traps extending over a few tens of nanometers from the GeSn/Ge interface into the Ge layer have therefore been considered. With a concentration of traps of $2 \times 10^{17} \mathrm{~cm}^{-3}$ extending up to $50 \mathrm{~nm}$ inside the Ge layer, the simulated Arrhenius plot then yields an activation energy that is similar to the experimentally extracted value (Fig. 3). Various energy positions of the traps within the band-gap have been tested, and traps located between $200 \mathrm{meV}$ and $250 \mathrm{meV}$ above the valence band give activation energies closest to the experimental activation energy. Given the small extent of the traps and their concentration that is higher than the Ge carrier concentration, these traps should therefore be interpreted as interface states at the GeSn/ Ge interface. These could also originate from the intermixing of GeSn and Ge, resulting in Sn-vacancy deep levels, which are expected to have an energy $190 \mathrm{meV}$ above the valence band[30].

One should note, however, that the value of the reverse current extracted from the linear part of the forward regime is located in the low range of the reverse currents experimentally observed. For the I-V characteristics at 280 $\mathrm{K}$, for instance, the extracted value $I_{S}$ is $1.9 \times 10^{-3} \mathrm{~A} \mathrm{~cm}^{-2}$ and at $250 \mathrm{~K}, I_{S}$ is $3.7 \times 10^{-4} \mathrm{~A} \mathrm{~cm}^{-2}$, whereas the values of the reverse current increase to $0.1 \mathrm{~A} \mathrm{~cm}^{-2}$ at $280 \mathrm{~K}$ and $4 \times 10^{-2} \mathrm{~A} \mathrm{~cm}^{-2}$ at $250 \mathrm{~K}$ and $-1 \mathrm{~V}$, respectively. This suggests that the extracted activation energy of $0.3 \mathrm{eV}$ is not the only mechanism leading to the measured total reverse current. Because of the strong bias dependence of the total reverse current, effects such as trap-assisted tunneling or even band-to-band tunneling can be expected[31].

Furthermore, the ideality factor has been calculated as a function of temperature and increases when the temperature decreases. The ideality factor is $n=1.4$ at $280 \mathrm{~K}$ and $n=2.8$ at $140 \mathrm{~K}$, and is larger than $n=2$ for temperatures lower than $180 \mathrm{~K}$. This indicates that the current is not limited by drift and diffusion or by recombination in the depletion region anymore, and would tend to confirm that other processes do occur in the reverse-bias regime. These processes are not included in our numerical model, but several suggestions have been made as to the origin of such large ideality factors, pointing again to effects such as trap-assisted tunneling or field-enhanced recombinations through isolated point defects[32].

\subsection{Capacitance-voltage characteristics}

Capacitance-voltage $(\mathrm{C}-\mathrm{V})$ measurements have been performed at various frequencies. Figure 4 (a) shows the re- 


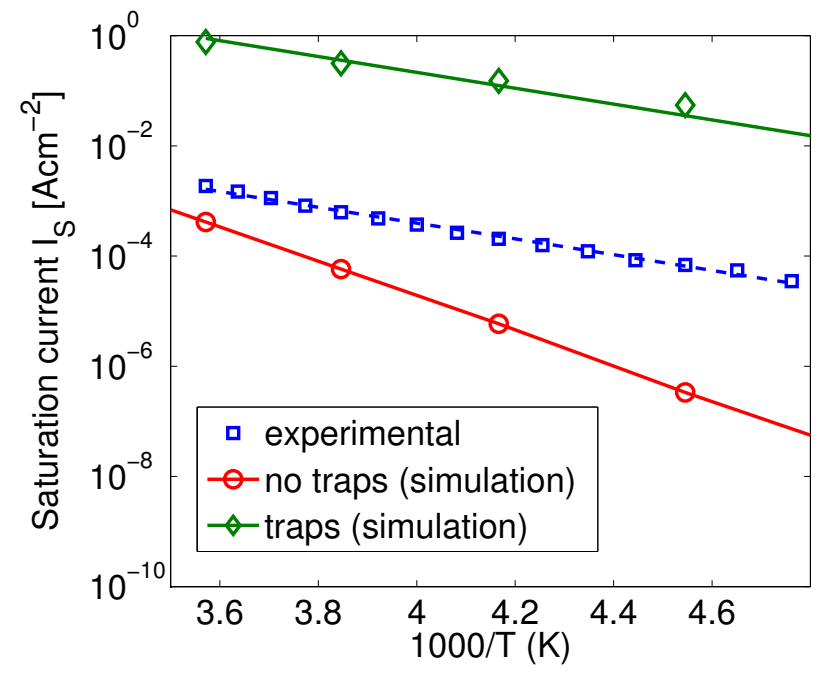

Figure 3: Extracted reverse saturation current as a function of the reciprocal temperature : experiment and simulations without defects and with defects extending $50 \mathrm{~nm}$ inside the Ge layer.

verse $\mathrm{C}-\mathrm{V}$ characteristics measured at three different frequencies. A weak frequency dispersion is observed, indicating that the traps present at the GeSn/Ge interface do not have a strong influence on the frequency characteristics. This is consistent with the fact that, when present in a narrow region near the interface, the contribution of these traps to the total capacitance is very limited with respect to the depletion capacitance and, therefore, does not alter the general behaviour of the capacitance as a function of applied bias.

A $1 / C^{2}$ analysis has been performed and shows a very linear behaviour, as is expected from a high-quality uniformlydoped material. Extraction of the active dopant concentration from the slopes of the $1 / C^{2}$ plots yields values ranging from $1.4 \times 10^{16} \mathrm{~cm}^{-3}$ to $2.4 \times 10^{16} \mathrm{~cm}^{-3}$. As this analysis gives access to the lowest concentration of active dopants in an asymmetric junction, the calculated value is therefore associated to the carrier concentration of the Ge substrate.

As shown in Fig. 4 (b), the behaviour of the simulated $\mathrm{C}-\mathrm{V}$ curves is similar to that of the experimental observations. The simulated values have been corrected to match the built-in potential of the experimental structure. This means that the quadratic behaviour of both measurements and simulations is identical for similar carrier concentrations, and so is the slope of $1 / C^{2}$. Only the $\mathrm{x}$-intercept is different from the experimental data. In the simulations, the addition of traps in the GeSn layer does not alter the slope of $1 / C^{2}$. Only when a high concentration of traps, $2 \times 10^{18} \mathrm{~cm}^{-3}$, is included in the Ge layer near the interface, does the slope begins to be significantly modified (Fig. 4 (b)).

In the simulations, the microscopic carrier concentration can also be observed as a function of position. The depletion width is controlled by the applied bias and extends mostly in the Ge substrate, as expected from the much lower doping of the Ge substrate. When a larger reverse bias is applied, the depletion region widens, and does so mostly on the Ge side of the interface. The total value of the simulated capacitance is also directly linked to the width of the depletion region, thereby confirming the previous $1 / C^{2}$ analysis to calculate the doping concentration of the Ge substrate.

Based on the simulated $\mathrm{C}-\mathrm{V}$ characteristics, we observed that, even though the $1 / C^{2}$ analysis only gives in- formation on the doping concentration of the Ge substrate, the value of the maximum of the capacitance, occuring for small forward biases, relates to the carrier concentration of the GeSn layer (Fig. 5 a). The maximum value of the capacitance in forward bias, occuring at different bias values depending on the GeSn layer carrier concentration, increases linearly with the carrier concentration in the GeSn layer, as shown in Fig. $5 \mathrm{~b}$.

The rate of increasing is dependent on the modulation frequency. For carrier concentrations similar to that of the Ge substrate, the relationship does not hold anymore (inset of Fig. $5 \mathrm{~b}$ ), as the maximum capacitance is influenced by carrier concentrations in both materials. Simulations give access to the amplitude of the local out-ofphase carrier concentration modulation. Integrating these amplitudes over the entire structure gives the total net charge modulation, which can be directly linked to the small-signal capacitance $C=d Q / d V$. Our calculations to determine the maximum charge modulation depending on the carrier concentration in GeSn confirmed the link previously observed with the maximum capacitance. 

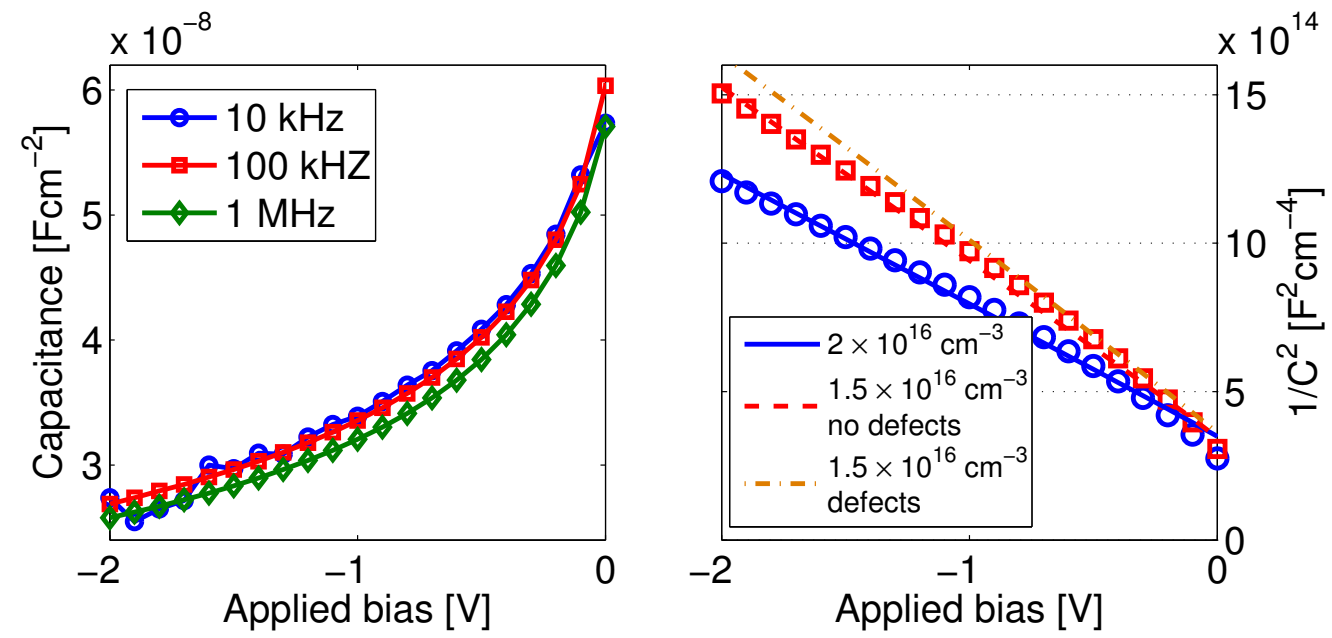

Figure 4: (a) Frequency dispersion of the reverse-bias C-V characteristics for a $300 \mu$ m diameter diode (b) Comparison of the experimental $1 / C^{2}$ for $150 \mu \mathrm{m}$ diameter (circles) and $300 \mu \mathrm{m}$ diameter (squares) and the simulated $1 / C^{2}$ reverse-bias capacitance for two values of the carrier concentrations in the Ge layer with or without the presence of traps at the interface.
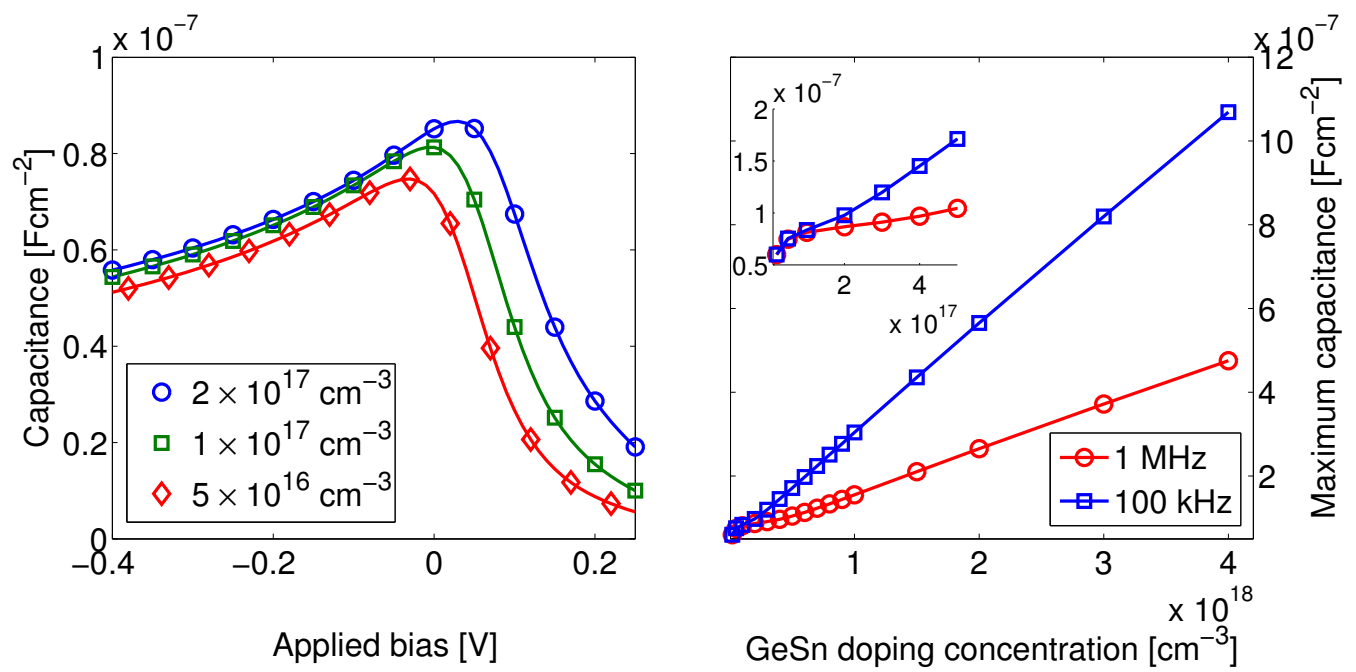

Figure 5: (a) Capacitance as function of the applied bias for carrier concentrations from $5 \times 10^{16} \mathrm{~cm}^{-3}$ to $2 \times 10^{17} \mathrm{~cm}^{-3}$ in the GeSn layer and a $1 \mathrm{MHz}$ frequency. (b) Maximum forward capacitance as a function of dopant concentration in the GeSn layer. Inset shows a magnification of the region of low concentration. 


\section{Conclusion}

Diodes consisting of the p-GeSn/n-Ge heterojunction with $5.8 \%$ Sn concentration and high Boron doping of the GeSn layer have been investigated. The measured electrical characteristics reveal good-quality diodes, with little effect of the presence of traps in the structure. An ideality factor at room temperature of 1.2 indicates limited recombinations occuring in the diodes, even though an activation energy of approximately $0.3 \mathrm{eV}$ is reported, indicating the presence of recombination centers in the space charge region. Strong bias-dependence of the reverse current denotes a field-enhanced mechanism such as trapassisted tunneling or even band-to-band tunneling. The carrier concentration in the Ge layer was determined by $\mathrm{C}-\mathrm{V}$ measurements while simulations show a link between the maximum of the capacitance at small forward bias and the dopant concentration in the GeSn layer.

\section{Acknowledgement}

Technical support from Microsys Lab of University of Liege is gratefully acknowledged. Financial supports by F.R.S.-FNRS (Crédit de recherche J.0119.14) and by Communauté française Wallonie-Bruxelles (Projet C-13/77) are also greatly acknowledged.

\section{References}

[1] M. Oehme, E. Kasper, J. Schulze, GeSn heterojunction diode: Detector and emitter in one device, ECS Journal of Solid State Science and Technology 2 (4) (2013) R76R78.

[2] H. H. Tseng, H. Li, V. Mashanov, Y. J. Yang, H. H. Cheng, G. E. Chang, R. A. Soref, G. Sun, GeSn-based p-i-n photodiodes with strained active layer on a si wafer, Applied Physics Letters 103 (23) (2013) 231907. doi:10.1063/1.4840135.

[3] S. Wirths, Z. Ikonic, A. Tiedemann, B. Holländer, T. Stoica, G. Mussler, U. Breuer, J. Hartmann, A. Benedetti, S. Chiussi, et al., Tensely strained GeSn alloys as optical gain media, Applied Physics Letters 103 (19) (2013) 192110.

[4] B. Vincent, Y. Shimura, S. Takeuchi, T. Nishimura, G. Eneman, A. Firrincieli, J. Demeulemeester, A. Vantomme, T. Clarysse, O. Nakatsuka, et al., Characterization of GeSn materials for future Ge pMOSFETs source/drain stressors, Microelectronic Engineering 88 (4) (2011) 342-346.

[5] S. Wirths, A. T. Tiedemann, Z. Ikonic, P. Harrison, B. Hollander, T. Stoica, G. Mussler, M. Myronov, J. M. Hartmann, D. Grutzmacher, D. Buca, S. Mantl, Band engineering and growth of tensile strained ge/(Si)GeSn heterostructures for tunnel field effect transistors, Applied Physics Letters 102 (19) (2013) 192103. doi:10.1063/1.4805034.

[6] G. Han, S. Su, Q. Zhou, P. Guo, Y. Yang, C. Zhan, L. Wang, W. Wang, Q. Wang, C. Xue, et al., Dopant Segregation and Nickel Stanogermanide Contact Formation on Source/Drain, Electron Device Letters, IEEE 33 (5) (2012) 634-636.

[7] R. Loo, B. Vincent, F. Gencarelli, C. Merckling, A. Kumar, G. Eneman, L. Witters, W. Vandervorst, M. Caymax, M. Heyns, Ge1-xSnx materials: Challenges and applications, ECS Journal of Solid State Science and Technology 2 (1) (2013) N35N40.

[8] S. Gupta, R. Chen, B. Vincent, D. Lin, B. Magyari-Kope, M. Caymax, J. Dekoster, J. S. Harris, Y. Nishi, K. Saraswat, GeSn channel $\mathrm{n}$ and $\mathrm{p}$ MOSFETs, in: Meeting Abstracts, The Electrochemical Society, 2012, p. 3222.
[9] X. Gong, G. Han, F. Bai, S. Su, P. Guo, Y. Yang, R. Cheng, D. Zhang, G. Zhang, C. Xue, B. Cheng, J. Pan, Z. Zhang, E. S. Tok, D. Antoniadis, Y.-C. Yeo, Germanium-tin (GeSn) pchannel MOSFETs fabricated on (100) and (111) surface orientations with sub-400C Si2H6 passivation, IEEE Electron Device Letters 34 (3) (2013) 339-341. doi:10.1109/LED.2012.2236880.

[10] S. Zaima, Technology evolution for silicon nanoelectronics: Postscaling technology, Japanese Journal of Applied Physics 52 (3R) (2013) 030001. doi:10.7567/JJAP.52.030001.

[11] E. A. Fitzgerald, N. Chand, Epitaxial Necking in GaAs Grown on Pre-Patterned Si Substrates, J. Electron. Mater. 20 (7) (1991) 839-853.

[12] N. D. Nguyen, G. Wang, G. Brammertz, M. Leys, N. Waldron, G. Winderickx, K. Lismont, J. Dekoster, R. Loo, M. Meuris, S. Degroote, F. Buttita, B. O'Neil, O. Feron, J. Lindner, F. Schulte, F. Schineller, M. Heuken, M. Caymax, Selective epitaxial growth of III-V semiconductor heterostructures on Si substrates for logic applications, ECS Transactions 33 (6) (2010) 933-939.

[13] G. Wang, N. D. Nguyen, M. R. Leys, R. Loo, G. Brammertz, O. Richard, H. Bender, J. Dekoster, M. Meuris, H. M. M, M. Caymax, Selective epitaxial growth of InP in STI trenches on off-axis Si (001) substrates, ECS Transactions 27 (1) (2010) 959-964.

[14] N. Waldron, N. D. Nguyen, T. Orzali, C. Merckling, G. Brammertz, P. Ong, G. Winderickx, G. Hellings, G. Eneman, M. Cay$\max$, M. Meuris, N. Horiguchi, A. Thean, Integration of InGaAs channel n-MOS devices on $200 \mathrm{~mm}$ Si wafers using the aspectratio-trapping technique, ECS Transactions 45 (4) (2012) 115128.

[15] B. Vincent, F. Gencarelli, H. Bender, C. Merckling, B. Douhard, D. Petersen, O. Hansen, H. Henrichsen, J. Meersschaut, W. Vandervorst, et al., Undoped and in-situ B doped GeSn epitaxial growth on Ge by atmospheric pressure-chemical vapor deposition, Appl. Phys. Lett. 99 (15) (2011) 152103-152103.

[16] F. Gencarelli, B. Vincent, L. Souriau, O. Richard, W. Vandervorst, R. Loo, M. Caymax, M. Heyns, Low-temperature Ge and GeSn Chemical Vapor Deposition using Ge2H6, Thin Solid Films 520 (8) (2012) 3211-3215.

[17] F. Gencarelli, B. Vincent, J. Demeulemeester, A. Vantomme, A. Moussa, A. Franquet, A. Kumar, H. Bender, J. Meersschaut, W. Vandervorst, et al., Crystalline properties and strain relaxation mechanism of CVD grown GeSn, ECS Journal of Solid State Science and Technology 2 (4) (2013) 134-137.

[18] B. Baert, O. Nakatsuka, S. Zaima, N. D. Nguyen, Impedance Spectroscopy of GeSn-based Heterostructures, ECS Transactions 50 (9) (2013) 481-490.

[19] S. Selberherr, Analysis and Simulation of Semiconductor Devices, Wiley, New York, 1981.

[20] N. D. Nguyen, M. Schmeits, Numerical simulation of impedance and admittance of OLEDs, Phys. Status Solidi (a) 203 (8) (2006) 1901-1914.

[21] B. Baert, M. Schmeits, N. Nguyen, Study of the energy distribution of the interface trap density in a GeSn MOS structure by numerical simulation of the electrical characteristics, Applied Surface Science 291 (2014) 25-30. doi:10.1016/j.apsusc.2013.09.022.

[22] M. Schmeits, N. D. Nguyen, M. Germain, Competition between deep impurity and dopant behavior of $\mathrm{Mg}$ in GaN Schottky diodes, J. Appl. Phys. 89 (3) (2001) 1890-1897.

[23] M. Sakhaf, M. Schmeits, Capacitance and conductance of semiconductor heterojunctions with continuous energy distribution of interface states, J. Appl. Phys. 80 (12) (1996) 6839-6848.

[24] Y. Shimura, W. Wang, T. Nieddu, F. Gencarelli, B. Vincent, P. Laha, H. Terryn, S. Stefanov, S. Chiussi, J. Van Campenhout, et al., Bandgap Measurement by Spectroscopic Ellipsometry for Strained Ge1-xSnx, 8th Int. Conf. on Si epitaxy and Heterostructures (ICSI-8), Book of Abstracts (2013) 65.

[25] J. Kouvetakis, J. Menendez, A. Chizmeshya, Tin-based group IV semiconductors: New platforms for opto-and microelectronics on silicon, Annu. Rev. Mater. Res. 36 (2006) 497-554. 
[26] A. Poyai, E. Simoen, C. Claeys, A. Czerwinski, E. Gaubas, Improved extraction of the activation energy of the leakage current in silicon $\mathrm{p}-\mathrm{n}$ junction diodes, Applied Physics Letters 78 (14) (2001) 1997-1999.

[27] A. Czerwinski, E. Simoen, A. Poyai, C. Claeys, Activation energy analysis as a tool for extraction and investigation of $\mathrm{p}-\mathrm{n}$ junction leakage current components, Journal of applied physics 94 (2) (2003) 1218-1221.

[28] R. Scheer, Activation energy of heterojunction diode currents in the limit of interface recombination, Journal of Applied Physics 105 (10) (2009) 104505-104505.

[29] S. M. Sze, K. K. Ng, Physics of semiconductor devices, John Wiley \& Sons, 2006.

[30] V. Markevich, A. Peaker, B. Hamilton, V. Litvinov, Y. M. Pokotilo, S. Lastovskii, J. Coutinho, A. Carvalho, M. Rayson, P. Briddon, Tin-vacancy complex in germanium, Journal of Applied Physics 109 (8) (2011) 083705.

[31] M. Gonzalez, E. Simoen, G. Eneman, B. D. Jaeger, G. Wang, R. Loo, C. Claeys, Defect assessment and leakage control in Ge junctions, Microelectronic Engineering 125 (0) (2014) 33 - 37. doi:http://dx.doi.org/10.1016/j.mee.2014.01.012.

[32] O. Breitenstein, J. Bauer, A. Lotnyk, J.-M. Wagner, Defect induced non-ideal dark i-v characteristics of solar cells, Superlattices and Microstructures 45 (4) (2009) 182-189. 